\title{
RECOVERY OF RESIDUAL FOREST ECOSYSTEM AS AN IMPACT OF SELECTIVE LOGGING IN SOUTH PAPUA: AN ECOLOGICAL APPROACH
}

\author{
AGUSTINUS MURDJOKO ${ }^{1 *}$, DJOKO MARSONO ${ }^{2}$, RONGGO SADONO $^{2}$ \\ and SUWARNO HADISUSANTO ${ }^{3}$ \\ ${ }^{1}$ Faculty of Forestry, Papua University, Manokwari 98314, Indonesia \\ ${ }^{2}$ Faculty of Forestry, Gadjah Mada University, Yogyakarta 55281, Indonesia \\ ${ }^{3}$ Faculty of Biology, Gadjah Mada University, Yogyakarta 55281, Indonesia \\ Received 7 December 2016/Accepted 3 June 2017
}

\begin{abstract}
Papua has been experiencing heavy logging activity in its forests for decades. However, only several studies focused on the effect of logging in the forest ecosystem. This research was aimed to analyze recovery processes of the forest ecosystem. The research was conducted in the logged tropical rainforest in South Papua using ecological approach which used tree communities as biotic and soil condition as abiotic indicators. Data were collected in the logging area of PT Tunas Timber Lestari located in the tropical rainforest of South Papua. There were five groups of forests used in this research i.e. unlogged, one year post selectively-logged, five years post selectively-logged, ten years post selectively-logged and fifteen years post selectively-logged forests. Thirty nested plots were laid on each forest group. Canonical Correspondence Analysis (CCA) was applied to analyze the understory and upperstory plant communities. Understory and upperstory plant communities formed different patterns due to logging. Plant communities in the ten and fifteen years post-selectively logged forests were not similar to those in the unlogged forest. Soil organic matter (SOM) content in the selectively logged forests was lower than that in the unlogged forest. These occurrences indicated that the selectively logged forests were still recovering and required more than fifteen years to be fully recovered.
\end{abstract}

Keywords: Canonical correspondence analysis, edaphic factor, logged tropical forest, plant community, soil organic matter

\section{INTRODUCTION}

Tropical rainforests play an important role in ecosystem services, such as logging production (Whitfeld et al. 2014; Putz \& Romero 2014). The process of production mechanism in the tropical rainforest has a significant impact on abiotic and biotic elements (Zambrano et al. 2014). Those conditions result in the change in the tropical rainforest as an ecosystem and some circumstances of the secondary successional process take place as a response to ecological alterations. Furthermore, most of the tropical rainforests are experiencing the alterations and the selective logging has a significant impact on

*Corresponding author: agustinus.murdjoko.papua@gmail.com ecological factors (Corrià-Ainslie et al. 2015; Flores et al. 2014). Hence, the logged tropical rainforests are counting on the ability of forest recovery itself. Most indicators to analyse forest recovery are based on tree density, basal area (Whitfeld et al. 2014; Rutten et al. 2015) and growth rate of residual trees (Do et al. 2016; Hoang et al. 2011; West et al. 2014; Sist et al. 2014; Susanty et al. 2015) in the logged forests. However, the recovery of disturbed forests should not only be considered based on sustainable timber production, but the ecological elements such as soil conditions and residual trees should also be taken into account as forest recovery indicators.

Some areas in lowland tropical forests in South Papua were intended as logging concession for decades (Kuswandi \& Murdjoko 2015; Murdjoko 
2013; Kuswandi 2014). Few studies concerning the effects of logging in Papua logged forests were conducted. Some studies focused only on damages, changes in basal area (Gandhi \& Mitlöhner 2014), population dynamics of remaining trees (Murdjoko 2013; Kuswandi \& Murdjoko 2015; Murdjoko et al. 2016b) and biomass stock change (Hendri et al. 2012). Therefore, it is necessary to analyze forest recovery using the ecological approach in South Papua. In this analysis, the primary forest was considered as a stable forest ecosystem (Pennington et al.2015).

Ecological approach took tree communities as biotic factors where many processes such as tree associations, ecological responses of the tree to ecological change as well as successional development can be analyzed based on patterns of tree communities. Besides that, soil condition alters after selective logging (Hattori et al. 2013) mainly the amount of soil properties decrease such as Nitrogen content (Asase et al. 2014), soil organic matter (SOM) (Prasetyo et al. 2015) and other nutrients (Duah-Gyamfi et al. 2014; Wasrin \& Putera 1999; Edwards et al. 2014; Imai et al.
2012). Consequently, the edaphic conditions were considered as abiotic indicators to support the explanation of the change in tree communities.

This research was aimed to analyze recovery process of selectively logged tropical rainforest ecosystem in South Papua using ecological approach. Our hypotheses were: 1. tree communities in a selectively logged tropical rainforest were considered to be recovered when tree communities in the rainforest were similar to those in the primary forest; 2 . the selectively logged tropical rainforest was considered to be recovered when the edaphic indicators in the rainforest were similar to those in the primary forest.

\section{MATERIALS AND METHODS}

\section{Study Area}

Research was conducted in the logging area of PT Tunas Timber Lestari located in the tropical rainforest of South Papua with geographical position between $140^{\circ} 21^{`}-140^{\circ} 59^{\prime} \mathrm{E}$ and $05^{\circ} 50^{\circ}$ $-06^{\circ} 42^{`} \mathrm{~S}$ (Fig.1). The annual rainfall was between

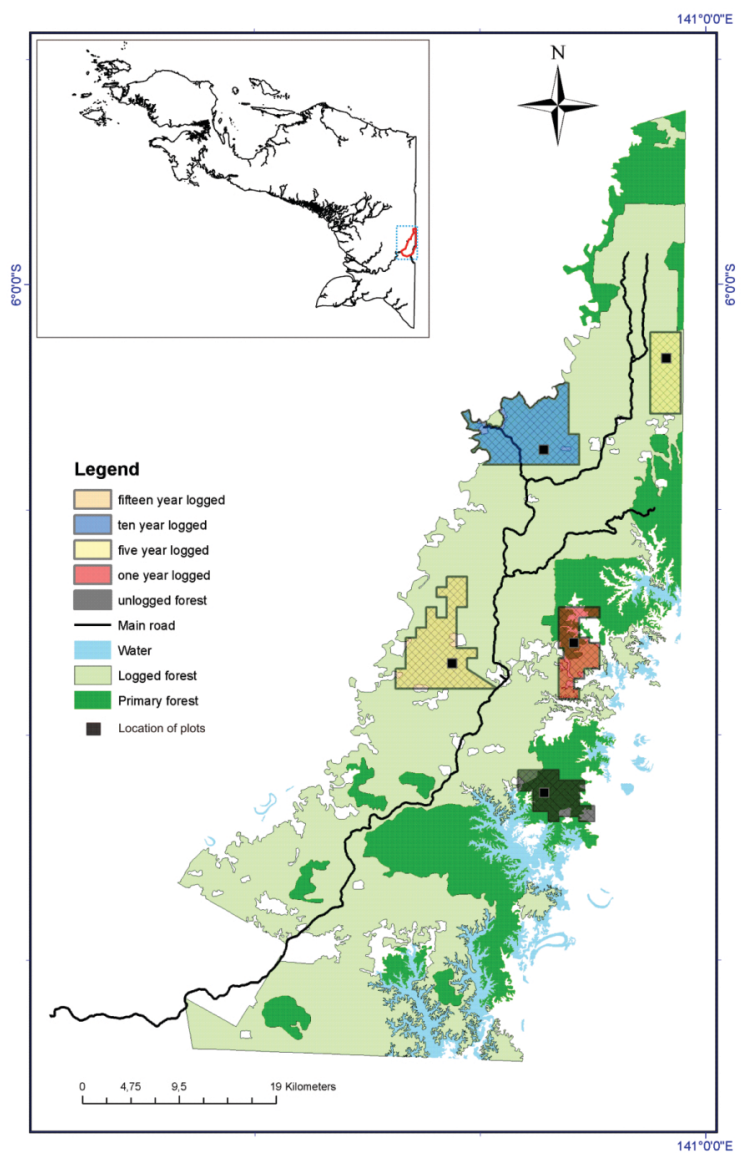

Figure 1 Study area in logging concession of PT Tunas Timber Lestari (Murdjoko et al. 2016c) 
3,000 and 4,000 $\mathrm{mm}$ with daily moisture range of $75-85 \%$. The edaphic condition was typified as lowland forest with almost flat topography with soil formed by alluvial process (Petocz 1989). The vegetation was dominated by trees belong to Dipterocarpaceae, Lauraceae and Myrtaceae families (Gandhi \& Mitlöhner 2014; Kuswandi et al. 2015). Several other plants such as lianas, rattans, ferns, palms, herbs, orchids and pandanus grew and interacted with trees in this forest (Murdjoko et al. 2016a).

Five groups of forests were used in this research i.e. unlogged, one year post selectivelylogged, five years post selectively-logged, ten years post selectively-logged and fifteen years post selectively-logged forests. The unlogged forest was taken as a primary forest which was a stable forest ecosystem. The selectively logged forests were compared to the unlogged forest to observe the recovery process. The selective logging was carried out by selectively cutting commercial trees having diameter of $\geq 40 \mathrm{~cm}$.

\section{Sampling and Data Collection}

Samples were collected in each forest group using systematic sampling plots. The first plot was placed at $200 \mathrm{~m}$ from the main road to avoid edge effect. The plots were rectangular with various sizes i.e. 1. $20 \times 20 \mathrm{~m}$ for trees (D) having DBH (diameter at breast height) of $\geq 20 \mathrm{~cm} ; 2$. $10 \times 10 \mathrm{~m}$ for poles $(\mathrm{C})$ having $\mathrm{DBH}$ of 10 to $<20$ $\mathrm{cm} ; 3.5 \times 5 \mathrm{~m}$ for saplings (B) having height of $>1.5 \mathrm{~m}$ and $\mathrm{DBH}$ of $<10 \mathrm{~cm}$; and $2 \times 2 \mathrm{~m}$ for seedlings (A) having height of $<1.5 \mathrm{~m}$. The four plots were set as nested plot (Fig. 2a). Thirty

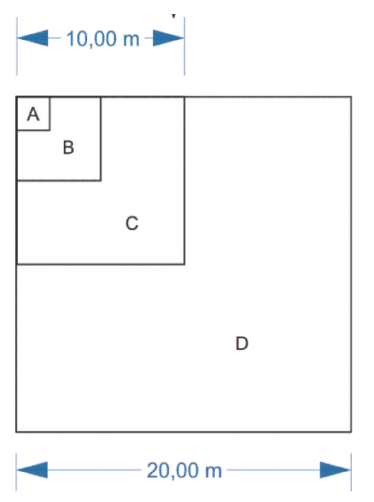

a

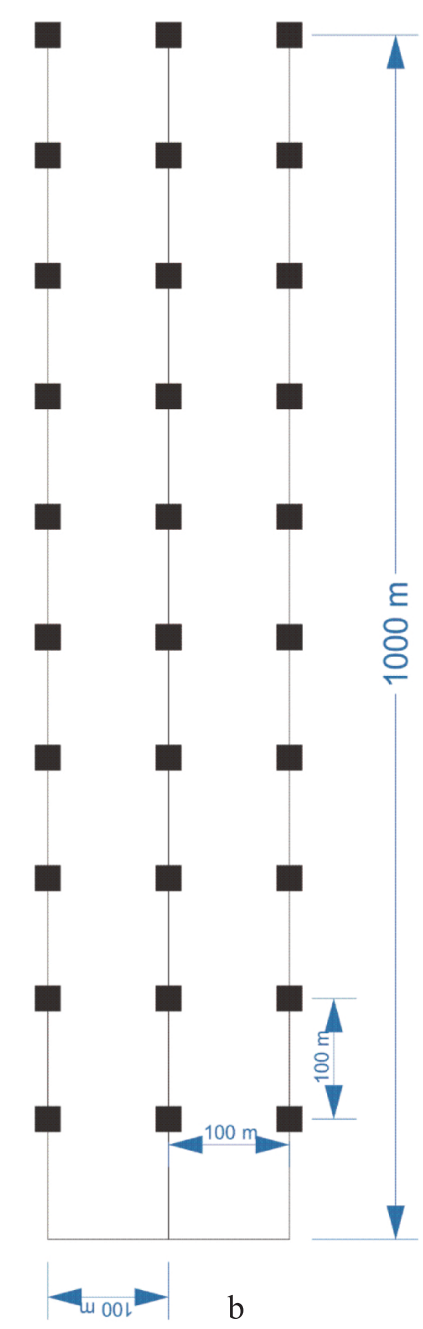

Figure 2 Nested plots to measure individual plant in both unlogged and selectively-logged forests

Note: $\mathrm{A}=$ plot for seedlings; $\mathrm{B}=$ plot for saplings; $\mathrm{C}=$ plot for for poles; $\mathrm{D}=$ plot for trees; (a) Distance between plots $=100 \mathrm{~m}$; (b) The 30 nested plots were laid on each forest group (unlogged, one year, five years, ten years and fifteen years post selectively-logged forests) 
nested plots were laid in each forest (Fig. 2b) making a total of 150 nested plots for the 5 forest groups (unlogged, one year, five years, ten years and fifteen years post selectively-logged forests). Seedlings and saplings were sampled as understory, while poles and trees were sampled as upperstory in both unlogged and selectively logged forests.

Data collected from seedlings, saplings, poles and trees consisted of numbers of individuals, diameter of individuals for those having $\mathrm{DBH} \geq$ $10 \mathrm{~cm}$ and species name of individuals. Species identification was carried out by two herbarium technicians. Unidentified samples were set as voucher specimens and sent to the herbarium of "Balai Penelitian dan Pengembangan Lingkungan Hidup dan Kebutanan (BP2LHK) Manokwari" and Herbarium Manokwariense (MAN) Pusat Penelitian Keanekaragaman Hayati Universitas Papua (PPKH-UNIPA), Manokwari. Validation of the species names of the individuals was checked online at http://www.theplantlist.org/; http://plants.jstor.org and www.ipni.org/ipni/.

Soil samples were taken from the center and four corners of the $20 \times 20 \mathrm{~m}$ plot. The litterfall samples were collected from each plot by making 1 $\mathrm{x} 1 \mathrm{~m}$ rectangular subplots in each plot. The soil and litterfall samples were sent to the laboratory of Balai Pengkajian Teknologi Pertanian Yogyakarta for determining the content of soil organic matter (SOM) for soil samples as well as Carbon (C) content, Nitrogen $(\mathrm{N})$ content and dry weight for litterfall samples.

\section{Data and Statistical Analysis}

Canonical Correspondence Analysis (CCA) was applied to show the relationship among tree species using stem density and environmental factors (SOM, C, N contents and dry weight of litterfall) (ter Braak 1987; ter Braak 1986; Khairil et al.2014). Plants communities were grouped as: a) understory consisted of small individuals (seedlings and saplings); and b) upperstory consisted of large individuals (poles and trees). Tree communities were formed as a result of interaction among tree species, SOM, C content, $\mathrm{N}$ content, dry weight of litterfall and forest groups (unlogged, one year, five years, ten years and fifteen years post selectively-logged). The CCA was computed using $\mathrm{R}$ statistical software version 3.3.1. with VEGAN package (R Core Team 2014; Oksanen et al. 2013). The tree communities were grouped using Euclidean distance among tree species. The Euclidean distance among tree communities was calculated as the average and confidence interval of $95 \%$.

\section{RESULTS AND DISCUSSION}

\section{Tree Communities}

Total tree species in the study area were 163 species and classified as understory (159 species) and upperstory (127 species) (Table 1). Within tree species, there were 106 species consisted of both understory and upperstory.

Table 1 Understory (a) and upperstory (b) tree communities formed due to logging activities

\begin{tabular}{|c|c|c|c|c|c|c|c|c|c|}
\hline \multicolumn{2}{|c|}{ No } & \multirow[b]{2}{*}{ Calophyllum peekelii Lauterb. } & \multirow{2}{*}{$\begin{array}{c}\text { Code } \\
\text { Calo_pe }\end{array}$} & \multirow{2}{*}{$\begin{array}{l}\mathrm{PF} \\
\sqrt{ }\end{array}$} & \multirow[t]{2}{*}{ X1LF } & \multirow[t]{2}{*}{ X5LF } & \multirow[t]{2}{*}{$\begin{array}{l}\text { X10LF } \\
\text { X15LF }\end{array}$} & \multirow[t]{2}{*}{ ALL } & \multirow[t]{2}{*}{ NON_AC } \\
\hline 1 & 1 & & & & & & & & \\
\hline 2 & 2 & Knema sp. & Knem_sp & $\sqrt{ }$ & & & & & \\
\hline 3 & 3 & Gonocaryum litorale (Blume) Sleumer & Gono_li & $\sqrt{ }$ & & & & & \\
\hline 4 & 4 & Alstonia scholaris (L.) R. Br. & Alst_sc & $\sqrt{ }$ & & & & & \\
\hline 5 & 5 & Guioa pleuropteris (Blume) Radlk. & Guio_pl & $\sqrt{ }$ & & & & & \\
\hline 6 & 6 & Dysoxylum sp. & Dyso_sp & $\sqrt{ }$ & & & & & \\
\hline 7 & 7 & Lepisanthes sp. & Lepi_sp & $\sqrt{ }$ & & & & & \\
\hline 8 & 8 & Rhodomyrtus sp. & Rhod_sp & $\sqrt{ }$ & & & & & \\
\hline 9 & 9 & Maasia glauca (Hassk.) Mols, Kessler \& Rogstad & Maas_gl & $\sqrt{ }$ & & & & & \\
\hline 10 & 10 & Octamyrtus sp. & Octa_sp & $\sqrt{ }$ & & & & & \\
\hline 11 & 11 & Chisocheton sp. & Chis_sp & $\sqrt{ }$ & & & & & \\
\hline 12 & 12 & Elaeocarpus ambemicus F.Muell. & Elae_ar & $\sqrt{ }$ & & & & & \\
\hline 13 & 13 & Haplolobus floribundus (K.Schum.) H.J.Lam & Hapl_fl & $\sqrt{ }$ & & & & & \\
\hline
\end{tabular}

Note: PF = unlogged forest; X1LF = one year post selectively-logged forest; X5LF = five years post selectively-logged forest; X10LF = ten years post selectively-logged forest; X15LF = fifteen years post selectively-logged forest; ALL = present in all forest groups; NON_AC $=$ not associated 
BIOTROPIA Vol. 24 No. 3, 2017

Table 1 Continued

\begin{tabular}{|c|c|c|c|c|c|c|c|c|c|}
\hline \multicolumn{2}{|c|}{ No } & \multirow[b]{2}{*}{ Brackenridgea sp. } & \multirow{2}{*}{$\frac{\text { Code }}{\text { Brac_sp.1 }}$} & \multirow{2}{*}{$\frac{\mathrm{PF}}{\sqrt{ }}$} & \multirow[t]{2}{*}{$\mathrm{X} 1 \mathrm{LF}$} & \multirow[t]{2}{*}{$\mathrm{X} 5 \mathrm{LF}$} & \multirow[t]{2}{*}{$\begin{array}{l}\text { X10LF } \\
\text { X15LF } \\
\end{array}$} & \multirow[t]{2}{*}{ ALL } & \multirow[t]{2}{*}{ NON_AC } \\
\hline 14 & 14 & & & & & & & & \\
\hline 15 & 15 & Litsea sp. & Lits_sp & $\sqrt{ }$ & & & & & \\
\hline 16 & 16 & Dysoxylum mollissimum Blume & Dyso_mo & $\sqrt{ }$ & & & & & \\
\hline 17 & 17 & Antiaris toxicaria Lesch. & Anti_to & $\sqrt{ }$ & & & & & \\
\hline 18 & 18 & Ficus variegata Blume & Ficu_va & $\sqrt{ }$ & & & & & \\
\hline 19 & 19 & Gyrinops versteegii (Gilg) Domke & Gyri_ve & $\sqrt{ }$ & & & & & \\
\hline 20 & 20 & Litsea guppyi (F. Muell.) F. Muell. ex Forman & Lits_gu & $\sqrt{ }$ & & & & & \\
\hline 21 & 21 & Maranthes corymbosa Blume & Mara_co & $\sqrt{ }$ & & & & & \\
\hline 22 & 22 & Mastixiodendron sp. & Mast_sp & $\sqrt{ }$ & & & & & \\
\hline 23 & 23 & V avaea amicorum Benth. & Vava_am & $\sqrt{ }$ & & & & & \\
\hline 24 & 24 & Calophyllum caudatum Kaneh. \& Hatus. & Calo_ca & $\sqrt{ }$ & & & & & \\
\hline 25 & 25 & Parastemon versteeghii Merr. \& L.M.Perry & Para_ve & $\sqrt{ }$ & & & & & \\
\hline 26 & 26 & Calophyllum laticostatum P.F.Stevens & Calo_la & $\sqrt{ }$ & & & & & \\
\hline 27 & 27 & Garcinia sp. & Garc_sp & $\sqrt{ }$ & & & & & \\
\hline 28 & 28 & Geniostoma sp. & Geni_sp & $\sqrt{ }$ & & & & & \\
\hline 29 & 1 & Sloanea pulchra (Schltr.) A.C.Sm. & Sloa_pu & & $\sqrt{ }$ & & & & \\
\hline 30 & 2 & Canarium sp. & Cana_sp & & $\sqrt{ }$ & & & & \\
\hline 31 & 3 & Horsfieldia sp. & Hors_sp & & $\sqrt{ }$ & & & & \\
\hline 32 & 4 & Melicope sp. & Meli_sp & & $\sqrt{ }$ & & & & \\
\hline 33 & 5 & Sterculia sp. & Ster_sp & & $\sqrt{ }$ & & & & \\
\hline 34 & 6 & Trema orientalis (L.) Blume & Trem_or & & $\sqrt{ }$ & & & & \\
\hline 35 & 7 & Trema sp. & Trem_sp & & $\sqrt{ }$ & & & & \\
\hline 36 & 8 & Trema tomentosa (Roxb.) H. Hara & Trem_to & & $\sqrt{ }$ & & & & \\
\hline 37 & 9 & Harpullia cupanioides Roxb. & Harp_cu & & $\sqrt{ }$ & & & & \\
\hline 38 & 10 & Sloanea sp. & Sloa_sp & & $\sqrt{ }$ & & & & \\
\hline 39 & 11 & Planchonella sp. & Plan_sp & & $\sqrt{ }$ & & & & \\
\hline 40 & 12 & Artabotrys sp. & Arta_sp & & $\sqrt{ }$ & & & & \\
\hline 41 & 13 & Arcbidendron parviflorum Pulle & Arch_pa & & $\sqrt{ }$ & & & & \\
\hline 42 & 14 & Elaeocarpus culminicola Warb. & Elae_cu & & $\sqrt{ }$ & & & & \\
\hline 43 & 15 & Diospyros papuana Valeton ex Bakh. & Dios_pa & & $\sqrt{ }$ & & & & \\
\hline 44 & 16 & Myristica globosa Warb. & Myri_gl & & $\sqrt{ }$ & & & & \\
\hline 45 & 17 & Glochidion sp. & Gloc_sp & & $\sqrt{ }$ & & & & \\
\hline 46 & 18 & Macaranga bifoveata J.J.Sm. & Maca_bi & & $\sqrt{ }$ & & & & \\
\hline 47 & 19 & Melicope elleryana (F. Muell.) T.G. Hartley & Meli_el & & $\sqrt{ }$ & & & & \\
\hline 48 & 20 & Kibara coriacea (Blume) Hook. f. \& A. Thomps. & Kiba_co & & $\sqrt{ }$ & & & & \\
\hline 49 & 21 & Timonius timon (Spreng.) Merr. & Timo_ti & & $\sqrt{ }$ & & & & \\
\hline 50 & 1 & Hopea papuana Diels & Hope_pa & & & $\sqrt{ }$ & & & \\
\hline 51 & 2 & Elaeocarpus angustifolius Blume & Elae_an & & & $\sqrt{ }$ & & & \\
\hline 52 & 3 & Ficus sp. & Ficu_sp & & & $\sqrt{ }$ & & & \\
\hline 53 & 4 & Ruta sp. & Ruta_sp & & & $\sqrt{ }$ & & & \\
\hline 54 & 5 & Garcinia latissima Miq. & Garc_la & & & $\sqrt{ }$ & & & \\
\hline 55 & 6 & Schefflera actinopbylla (Endl.) Harms & Sche_ac & & & $\sqrt{ }$ & & & \\
\hline 56 & 7 & Campnosperma brevipetiolatum Volkens & Camp_br & & & $\sqrt{ }$ & & & \\
\hline 57 & 8 & Goniothalamus sp. & Goni_sp & & & $\sqrt{ }$ & & & \\
\hline 58 & 9 & Corynocarpus laevigatus J.R.Forst. \& G.Forst. & Cory_la & & & $\sqrt{ }$ & & & \\
\hline 59 & 10 & Adenanthera pavonina $\mathrm{L}$. & Aden_pa & & & $\sqrt{ }$ & & & \\
\hline 60 & 11 & Aglaia spectabilis (Miq.) S.S.Jain \& S.Bennet & Agla_sp & & & $\sqrt{ }$ & & & \\
\hline 61 & 12 & Dillenia alata (R.Br. ex DC.) Banks ex Martelli & Dill_al & & & $\sqrt{ }$ & & & \\
\hline 62 & 13 & Dillenia indica $\mathrm{L}$. & Dill_in & & & $\sqrt{ }$ & & & \\
\hline 63 & 14 & Diospyros sp. & Dios_sp & & & $\sqrt{ }$ & & & \\
\hline 64 & 15 & Fagraea sp. & Fagr_sp & & & $\sqrt{ }$ & & & \\
\hline 65 & 16 & Flindersia pimenteliana F.Muell. & Flin_pi & & & $\sqrt{ }$ & & & \\
\hline 66 & 17 & Gynotroches sp. & Gyno_sp & & & $\sqrt{ }$ & & & \\
\hline 67 & 18 & Manilkara fasciculata (Warb.) H.J.Lam \& Maas Geest. & Mani_fa & & & $\sqrt{ }$ & & & \\
\hline
\end{tabular}


Table 1 Continued

\begin{tabular}{|c|c|c|c|c|c|c|c|c|c|}
\hline \multicolumn{2}{|c|}{ No } & \multirow{2}{*}{ Melicope bonwickii (F. Muell.) T.G. Hartley } & \multirow{2}{*}{ Code } & \multirow[t]{2}{*}{$\mathrm{PF}$} & \multirow[t]{2}{*}{$\mathrm{X} 1 \mathrm{LF}$} & \multirow{2}{*}{$\frac{\mathrm{X} 5 \mathrm{LF}}{\sqrt{ }}$} & \multirow[t]{2}{*}{$\begin{array}{l}\text { X10LF } \\
\text { X15LF }\end{array}$} & \multirow[t]{2}{*}{ ALL } & \multirow[t]{2}{*}{ NON_AC } \\
\hline 68 & 19 & & & & & & & & \\
\hline 69 & 20 & Prunus sp. & Prun_sp & & & $\sqrt{ }$ & & & \\
\hline 70 & 21 & Santiria sp. & Sant_sp & & & $\sqrt{ }$ & & & \\
\hline 71 & 1 & Prunus javanica (Teijsm. \& Binn.) Miq. & Prun_ja & & & & $\sqrt{ }$ & & \\
\hline 72 & 2 & Terminalia complanata K.Schum. & Term_co & & & & $\sqrt{ }$ & & \\
\hline 73 & 3 & Diospyros calycantha O.Schwarz & Dios_ca & & & & $\sqrt{ }$ & & \\
\hline 74 & 4 & Lithocarpus rufovillosus (Markgr.) Rehder & Lith_ru & & & & $\sqrt{ }$ & & \\
\hline 75 & 5 & Pisonia grandis $\mathrm{R}$. Br. & Piso_gr & & & & $\sqrt{ }$ & & \\
\hline 76 & 6 & Horsfieldia irya (Gaertn.) Warb. & Hors_ir & & & & $\sqrt{ }$ & & \\
\hline 77 & 7 & Cananga odorata (Lam.) Hook.f. \& Thomson & Cana_od & & & & $\sqrt{ }$ & & \\
\hline 78 & 8 & Carrierea sp. & Carr_sp & & & & $\sqrt{ }$ & & \\
\hline 79 & 9 & Lepisanthes rubiginosa (Roxb.) Leenh. & Lepi_ru & & & & $\sqrt{ }$ & & \\
\hline 80 & 10 & Mammea novoguineensis (Kan. \& Hat.) Kosterm. & Mamm_no & & & & $\sqrt{ }$ & & \\
\hline 81 & 11 & Pometia pinnata J.R.Forst. \& G.Forst. & Pome_pi & & & & $\sqrt{ }$ & & \\
\hline 82 & 12 & Semecarpus rufovelutinus Ridl. & Seme_ru & & & & $\sqrt{ }$ & & \\
\hline 83 & 13 & Siphonodon sp. & Siph_sp & & & & $\sqrt{ }$ & & \\
\hline 84 & 14 & Gluta papuana Ding Hou & Glut_pa & & & & $\sqrt{ }$ & & \\
\hline 85 & 15 & Prainea limpato (Miq.) Beumee ex K.Heyne & Prai_li & & & & $\sqrt{ }$ & & \\
\hline 86 & 16 & Maniltoa browneoides Harms & Mani_br & & & & $\sqrt{ }$ & & \\
\hline 87 & 17 & Jagera javanica (Blume) Kalkman & Jage_ja & & & & $\sqrt{ }$ & & \\
\hline 88 & 1 & Canarium birsutum Willd. & Cana_hi & & & & & $\sqrt{ }$ & \\
\hline 89 & 2 & Polyalthia sp. & Poly_sp & & & & & & $\sqrt{ }$ \\
\hline 90 & 3 & Virola surinamensis (Rol. ex Rottb.) Warb. & Viro_su & & & & & & $\sqrt{ }$ \\
\hline 91 & 4 & $\begin{array}{l}\text { Planchonella anteridifera (C.T.White \& W.D.Francis ex Lane-Poole) } \\
\text { H.J.Lam }\end{array}$ & Plan_an & & & & & & $\sqrt{ }$ \\
\hline 92 & 5 & Dracontomelon dao (Blanco) Merr. \& Rolfe & Drac_da & & & & & & $\sqrt{ }$ \\
\hline 93 & 6 & Magnolia tsiampacca (L.) Figlar \& Noot. & Magn_ts & & & & & & $\sqrt{ }$ \\
\hline 94 & 7 & Actinodaphne nitida Teschner & Acti_ni & & & & & & $\sqrt{ }$ \\
\hline 95 & 8 & Semecarpus papuana Lauterb. & Seme_pa & & & & & & $\sqrt{ }$ \\
\hline 96 & 9 & Planchonella keyensis H.J.Lam & Plan_ke & & & & & & $\sqrt{ }$ \\
\hline 97 & 10 & Sysygium anomalum Lauterb. & Syzy_an & & & & & & $\sqrt{ }$ \\
\hline 98 & 11 & Cleistanthus oblongifolius (Roxb.) Müll.Arg. & Clei_ob & & & & & & $\sqrt{ }$ \\
\hline 99 & 12 & Homalium foetidum Benth & Homa_fo & & & & & & $\sqrt{ }$ \\
\hline 100 & 13 & Popowia sp. & Popo_sp & & & & & & $\sqrt{ }$ \\
\hline 101 & 14 & Canarium indicum $\mathrm{L}$. & Cana_in & & & & & & $\sqrt{ }$ \\
\hline 102 & 15 & Pimelodendron amboinicum Hassk. & Pime_am & & & & & & $\sqrt{ }$ \\
\hline 103 & 16 & Blumeodendron tokbrai (Blume) Kurz & Blum_to & & & & & & $\sqrt{ }$ \\
\hline 104 & 17 & Aglaia argentea Blume & Agla_ar & & & & & & $\sqrt{ }$ \\
\hline 105 & 18 & Gnetum gnemon L. & Gnet_gn & & & & & & $\sqrt{ }$ \\
\hline 106 & 19 & Mammea sp. & Mamm_sp & & & & & & $\sqrt{ }$ \\
\hline 107 & 20 & Vatica rassak. Blume & Vati_ra & & & & & & $\sqrt{ }$ \\
\hline 108 & 21 & Fagraea racemosa Jack & Fagr_ra & & & & & & $\sqrt{ }$ \\
\hline 109 & 22 & Sterculia shillinglawii F.Muell. & Ster_sh & & & & & & $\sqrt{ }$ \\
\hline 110 & 23 & Neolitsea sp. & Neol_sp & & & & & & $\sqrt{ }$ \\
\hline 111 & 24 & Elaeocarpus sp. & Elae_sp & & & & & & $\sqrt{ }$ \\
\hline 112 & 25 & Endiandra rubescens (Blume) Miq. & Endi_ru & & & & & & $\sqrt{ }$ \\
\hline 113 & 26 & Endiandra sp. & Endi_sp & & & & & & $\sqrt{ }$ \\
\hline 114 & 27 & Hopea iriana Slooten & Hope_ir & & & & & & $\sqrt{ }$ \\
\hline 115 & 28 & Prunus arborea (Blume) Kalkman & Prun_ar & & & & & & $\sqrt{ }$ \\
\hline 116 & 29 & Lasianthus sp. & Lasi_sp & & & & & & $\sqrt{ }$ \\
\hline 117 & 30 & Terminalia copelandi Elmer & Term_co.1 & & & & & & $\sqrt{ }$ \\
\hline 118 & 31 & Sundacarpus amarus (Blume) C.N.Page & Sund_am & & & & & & $\sqrt{ }$ \\
\hline 119 & 32 & Chisocheton ceramicus Miq. & Chis_ce & & & & & & $\sqrt{ }$ \\
\hline 120 & 33 & Teijsmanniodendron bogoriense Koord. & Teij_bo & & & & & & $\sqrt{ }$ \\
\hline
\end{tabular}


BIOTROPIA Vol. 24 No. 3, 2017

Table 1 Continued

\begin{tabular}{|c|c|c|c|c|c|c|c|c|c|}
\hline \multicolumn{2}{|c|}{ No } & \multirow{2}{*}{$\begin{array}{c}\text { Species } \\
\text { Sloanea pullei O.C.Schmidt ex A.C.Sm. }\end{array}$} & \multirow{2}{*}{$\begin{array}{l}\text { Code } \\
\text { Sloa_pu.1 }\end{array}$} & \multirow[t]{2}{*}{$\mathrm{PF}$} & \multirow[t]{2}{*}{ X1LF } & \multirow[t]{2}{*}{$\mathrm{X} 5 \mathrm{LF}$} & \multirow[t]{2}{*}{$\begin{array}{l}\text { X10LF } \\
\text { X15LF }\end{array}$} & \multirow{2}{*}{$\frac{\text { ALL }}{\sqrt{ }}$} & \multirow[t]{2}{*}{ NON_AC } \\
\hline 121 & 34 & & & & & & & & \\
\hline 122 & 35 & Maasia sumatrana (Miq.) Mols, Kessler \& Rogstad & Maas_su & & & & & $\sqrt{ }$ & \\
\hline 123 & 36 & Cynometra ramiflora $\mathrm{L}$. & Cyno_ra & & & & & $\sqrt{ }$ & \\
\hline 124 & 37 & Canarium asperum Benth. & Cana_as & & & & & $\sqrt{ }$ & \\
\hline 125 & 38 & Alstonia spectabilis $\mathrm{R} . \mathrm{Br}$. & Alst_sp & & & & & $\sqrt{ }$ & \\
\hline 126 & 39 & Gymnacranthera farqubariana (Hook.f. \& Thomson) Warb. & Gymn_fa & & & & & $\sqrt{ }$ & \\
\hline 127 & 40 & Grewia sp. & Grew_sp & & & & & $\sqrt{ }$ & \\
\hline 128 & 41 & Pometia acuminata Radlk. & Pome_ac & & & & & $\sqrt{ }$ & \\
\hline 129 & 42 & Halfordia kendack Guillaumin & Half_ke & & & & & $\sqrt{ }$ & \\
\hline 130 & 43 & Timonius rufescens (Miq.) Boerl. & Timo_ru & & & & & $\sqrt{ }$ & \\
\hline 131 & 44 & Siphonodon celastrineus Griff. & Siph_ce & & & & & $\sqrt{ }$ & \\
\hline 132 & 45 & Palaquium lobbianum Burck & Pala_lo & & & & & $\sqrt{ }$ & \\
\hline 133 & 46 & Grewia eriocarpa Juss. & Grew_er & & & & & $\sqrt{ }$ & \\
\hline 134 & 47 & Gynotroches axillaris Blume & Gyno_ax & & & & & $\sqrt{ }$ & \\
\hline 135 & 48 & Planchonia careya (F.Muell.) R.Knuth & Plan_ca & & & & & $\sqrt{ }$ & \\
\hline 136 & 49 & Myristica sp. & Myri_sp & & & & & $\sqrt{ }$ & \\
\hline 137 & 50 & Garcinia picrorbiza Miq. & Garc_pi & & & & & $\sqrt{ }$ & \\
\hline 138 & 51 & Gironniera subaequalis Planch. & Giro_su & & & & & $\sqrt{ }$ & \\
\hline 139 & 52 & Buchanania arborescens (Blume) Blume & Buch_ar & & & & & $\sqrt{ }$ & \\
\hline 140 & 53 & Hopea celtidifolia Kosterm. & Hope_ce & & & & & $\sqrt{ }$ & \\
\hline 141 & 54 & Endospermum medullosum L.S.Sm. & Endo_me & & & & & $\sqrt{ }$ & \\
\hline 142 & 55 & Rhodamnia cinerea Jack & Rhod_ci & & & & & $\sqrt{ }$ & \\
\hline 143 & 1 & Adenanthera novo-guineensis Baker $\mathrm{f}$. & Aden_no & & & & & & $\sqrt{ }$ \\
\hline 144 & 2 & Anisoptera thurifera subsp. polyandra (Blume) P.S.Ashton & Anis_th & & & & & & $\sqrt{ }$ \\
\hline 145 & 3 & Brachychiton sp. & Brac_sp & & & & & & $\sqrt{ }$ \\
\hline 146 & 4 & Calophyllum sp. & Calo_sp & & & & & & $\sqrt{ }$ \\
\hline 147 & 5 & Carallia brachiata (Lour.) Merr. & Cara_br & & & & & & $\sqrt{ }$ \\
\hline 148 & 6 & Celtis latifolia (Blume) Planch. & Celt_la & & & & & & $\sqrt{ }$ \\
\hline 149 & 7 & Cerbera floribunda K.Schum. & Cerb_fl & & & & & & $\sqrt{ }$ \\
\hline 150 & 8 & Diospyros pilosanthera Blanco & Dios_pi & & & & & & $\sqrt{ }$ \\
\hline 151 & 9 & Garcinia dulcis (Roxb.) Kurz & Garc_du & & & & & & $\sqrt{ }$ \\
\hline 152 & 10 & Maniltoa plurijuga Merr. \& L.M.Perry & Mani_pl & & & & & & $\sqrt{ }$ \\
\hline 153 & 11 & Nageia wallichiana (C.Presl) Kuntze & Nage_wa & & & & & & $\sqrt{ }$ \\
\hline 154 & 12 & Santiria rubiginosa Blume & Sant_ru & & & & & & $\sqrt{ }$ \\
\hline 155 & 13 & Schizomeria katastega Mattf. & Schi_ka & & & & & & $\sqrt{ }$ \\
\hline 156 & 14 & Spatbiostemon javensis Blume & Spat_ja & & & & & & $\sqrt{ }$ \\
\hline 157 & 15 & Sterculia macropbylla Vent. & Ster_ma & & & & & & $\sqrt{ }$ \\
\hline 158 & 16 & Terminalia sp. & Term_sp & & & & & & $\sqrt{ }$ \\
\hline 159 & 17 & Vavaea sp. & Vava_sp & & & & & & $\sqrt{ }$ \\
\hline
\end{tabular}

b. Upperstory

\begin{tabular}{|c|c|c|c|c|c|c|c|c|c|}
\hline No & & Species & Code & $\mathrm{PF}$ & X1LF & X5LF & $\begin{array}{l}\text { X10LF } \\
\text { X15LF }\end{array}$ & ALL & NON_AC \\
\hline 1 & 1 & Terminalia complanata K.Schum. & Term_co & $\sqrt{ }$ & & & & & \\
\hline 2 & 2 & Siphonodon celastrineus Griff. & Siph_ce & $\sqrt{ }$ & & & & & \\
\hline 3 & 3 & Lepisanthes sp. & Lepi_sp & $\sqrt{ }$ & & & & & \\
\hline 4 & 4 & Rhodomyrtus sp. & Rhod_sp & $\sqrt{ }$ & & & & & \\
\hline 5 & 5 & Garcinia latissima Miq. & Garc_la & $\sqrt{ }$ & & & & & \\
\hline 6 & 6 & Alphitonia incana (Roxb.) Teijsm. \& Binn. ex Kurz & Alph_in & $\sqrt{ }$ & & & & & \\
\hline 7 & 7 & Dysoxylum sp. & Dyso_sp & $\sqrt{ }$ & & & & & \\
\hline 8 & 8 & Fagraea racemosa Jack & Fagr_ra & $\sqrt{ }$ & & & & & \\
\hline 9 & 9 & Flacourtia inermis Roxb. & Flac_in & $\sqrt{ }$ & & & & & \\
\hline 10 & 10 & Guioa pleuropteris (Blume) Radlk. & Guio_pl & $\sqrt{ }$ & & & & & \\
\hline 11 & 11 & Hopea papuana Diels & Hope_pa & $\sqrt{ }$ & & & & & \\
\hline
\end{tabular}


Recovery of residual forest ecosystem: impact of selective logging - Murdjoko et al.

Table 1 Continued

\begin{tabular}{|c|c|c|c|c|c|c|c|c|c|}
\hline \multicolumn{2}{|c|}{ No } & \multirow[b]{2}{*}{ Litsea timoriana Span. } & \multirow{2}{*}{$\frac{\text { Code }}{\text { Lits_ti }}$} & \multirow{2}{*}{$\frac{\mathrm{PF}}{\sqrt{ }}$} & \multirow[t]{2}{*}{$\mathrm{X} 1 \mathrm{LF}$} & \multirow[t]{2}{*}{ X5LF } & \multirow[t]{2}{*}{$\begin{array}{l}\text { X10LF } \\
\text { X15LF } \\
\end{array}$} & \multirow[t]{2}{*}{ ALL } & \multirow[t]{2}{*}{ NON_AC } \\
\hline 12 & 12 & & & & & & & & \\
\hline 13 & 13 & Nauclea orientalis (L.) L. & Nauc_or & $\sqrt{ }$ & & & & & \\
\hline 14 & 14 & Calophyllum laticostatum P.F.Stevens & Calo_la & $\sqrt{ }$ & & & & & \\
\hline 15 & 15 & Myristica globosa Warb. & Myri_gl & $\sqrt{ }$ & & & & & \\
\hline 16 & 16 & Octomeles sumatrana Miq. & Octo_su & $\sqrt{ }$ & & & & & \\
\hline 17 & 1 & Trema sp. & Trem_sp & & $\sqrt{ }$ & & & & \\
\hline 18 & 2 & Gonocaryum litorale (Blume) Sleumer & Gono_li & & $\sqrt{ }$ & & & & \\
\hline 19 & 3 & Kibara coriacea (Blume) Hook. f. \& A. Thomps. & Kiba_co & & $\sqrt{ }$ & & & & \\
\hline 20 & 4 & Canarium sp. & Cana_sp & & $\sqrt{ }$ & & & & \\
\hline 21 & 5 & Garcinia picrorhiza Miq. & Garc_pi & & $\sqrt{ }$ & & & & \\
\hline 22 & 6 & Dysoxylum mollissimum Blume & Dyso_mo & & $\sqrt{ }$ & & & & \\
\hline 23 & 7 & Rhodamnia cinerea Jack & Rhod_ci & & $\sqrt{ }$ & & & & \\
\hline 24 & 8 & Garcinia dulcis (Roxb.) Kurz & Garc_du & & $\sqrt{ }$ & & & & \\
\hline 25 & 9 & Calophyllum sp. & Calo_sp & & $\sqrt{ }$ & & & & \\
\hline 26 & 1 & Aglaia spectabilis (Miq.) S.S.Jain \& S.Bennet & Agla_sp & & & $\sqrt{ }$ & & & \\
\hline 27 & 2 & Brackenridgea sp. & Brac_sp & & & $\sqrt{ }$ & & & \\
\hline 28 & 3 & Elaeocarpus culminicola Warb. & Elae_cu & & & $\sqrt{ }$ & & & \\
\hline 29 & 4 & Fagraea sp. & Fagr_sp & & & $\sqrt{ }$ & & & \\
\hline 30 & 5 & Flindersia amboinensis Poir. & Flin_am & & & $\sqrt{ }$ & & & \\
\hline 31 & 6 & Planchonella densinervia (K.Krause) H.J.Lam & Plan_de & & & $\sqrt{ }$ & & & \\
\hline 32 & 7 & Terminalia sp. & Term_sp & & & $\sqrt{ }$ & & & \\
\hline 33 & 8 & Sloanea sp. & Sloa_sp & & & $\sqrt{ }$ & & & \\
\hline 34 & 9 & Teijsmanniodendron bogoriense Koord. & Teij_bo & & & $\sqrt{ }$ & & & \\
\hline 35 & 10 & Canarium indicum $\mathrm{L}$. & Cana_in & & & $\sqrt{ }$ & & & \\
\hline 36 & 11 & Buchanania arborescens (Blume) Blume & Buch_ar & & & $\sqrt{ }$ & & & \\
\hline 37 & 12 & Elaeocarpus angustifolius Blume & Elae_an & & & $\sqrt{ }$ & & & \\
\hline 38 & 13 & Prunus arborea (Blume) Kalkman & Prun_ar & & & $\sqrt{ }$ & & & \\
\hline 39 & 14 & Macaranga bifoveata J.J.Sm. & Maca_bi & & & $\sqrt{ }$ & & & \\
\hline 40 & 15 & Myristica sp. & Myri_sp & & & $\sqrt{ }$ & & & \\
\hline 41 & 16 & Magnolia tsiampacca (L.) Figlar \& Noot. & Magn_ts & & & $\sqrt{ }$ & & & \\
\hline 42 & 17 & Maasia glauca (Hassk.) Mols, Kessler \& Rogstad & Maas_gl & & & $\sqrt{ }$ & & & \\
\hline 43 & 18 & Manilkeara fasciculata (Warb.) H.J.Lam \& Maas Geest. & Mani_fa & & & $\sqrt{ }$ & & & \\
\hline 44 & 19 & Adenanthera pavonina $\mathrm{L}$. & Aden_pa & & & $\sqrt{ }$ & & & \\
\hline 45 & 20 & Alstonia scholaris (L.) R. Br. & Alst_sc & & & $\sqrt{ }$ & & & \\
\hline 46 & 21 & Breonia chinensis (Lam.) Capuron & Breo_ch & & & $\sqrt{ }$ & & & \\
\hline 47 & 22 & Corynocarpus laevigatus J.R.Forst. \& G.Forst. & Cory_la & & & $\sqrt{ }$ & & & \\
\hline 48 & 23 & Dillenia indica $\mathrm{L}$. & Dill_in & & & $\sqrt{ }$ & & & \\
\hline 49 & 24 & Diospyros pilosanthera Blanco & Dios_pi & & & $\sqrt{ }$ & & & \\
\hline 50 & 25 & Geniostoma sp. & Geni_sp & & & $\sqrt{ }$ & & & \\
\hline 51 & 26 & Maasia sumatrana (Miq.) Mols, Kessler \& Rogstad & Maas_su & & & $\sqrt{ }$ & & & \\
\hline 52 & 27 & Ochrosia sp. & Ochr_sp & & & $\sqrt{ }$ & & & \\
\hline 53 & 28 & Planchonella sp. & Plan_sp & & & $\sqrt{ }$ & & & \\
\hline 54 & 29 & Siphonodon sp. & Siph_sp & & & $\sqrt{ }$ & & & \\
\hline 55 & 30 & Syzygium acutangulum Nied. & Syzy_ac & & & $\sqrt{ }$ & & & \\
\hline 56 & 31 & Timonius rufescens (Miq.) Boerl. & Timo_ru & & & $\sqrt{ }$ & & & \\
\hline 57 & 32 & Actinodaphne nitida Teschner & Acti_ni & & & $\sqrt{ }$ & & & \\
\hline 58 & 33 & Haplolobus floribundus (K.Schum.) H.J.Lam & Hapl_fl & & & $\sqrt{ }$ & & & \\
\hline 59 & 34 & Mammea sp. & Mamm_sp & & & $\sqrt{ }$ & & & \\
\hline 60 & 1 & Aglaia argentea Blume & Agla_ar & & & & $\sqrt{ }$ & & \\
\hline 61 & 2 & Palaquium lobbianum Burck & Pala_lo & & & & $\sqrt{ }$ & & \\
\hline 62 & 3 & Gnetum gnemon L. & Gnet_gn & & & & $\sqrt{ }$ & & \\
\hline 63 & 4 & Maranthes corymbosa Blume & Mara_co & & & & $\sqrt{ }$ & & \\
\hline 64 & 5 & Polyaltbia sp. & Poly_sp & & & & $\sqrt{ }$ & & \\
\hline
\end{tabular}


BIOTROPIA Vol. 24 No. 3, 2017

Table 1 Continued

\begin{tabular}{|c|c|c|c|c|c|c|c|c|c|}
\hline \multicolumn{2}{|c|}{ No } & Species & \multirow{2}{*}{$\frac{\text { Code }}{\text { Flin_pi }}$} & \multirow[t]{2}{*}{$\mathrm{PF}$} & \multirow[t]{2}{*}{$\mathrm{X} 1 \mathrm{LF}$} & \multirow[t]{2}{*}{$\mathrm{X} 5 \mathrm{LF}$} & \multirow{2}{*}{$\begin{array}{c}\text { X10LF } \\
\text { X15LF } \\
\sqrt{ }\end{array}$} & \multirow[t]{2}{*}{ ALL } & \multirow[t]{2}{*}{ NON_AC } \\
\hline 65 & 6 & Flindersia pimenteliana F.Muell. & & & & & & & \\
\hline 66 & 7 & Maniltoa browneoides Harms & Mani_br & & & & $\sqrt{ }$ & & \\
\hline 67 & 8 & Chisocheton sp. & Chis_sp & & & & $\sqrt{ }$ & & \\
\hline 68 & 9 & Chisocheton ceramicus Miq. & Chis_ce & & & & $\sqrt{ }$ & & \\
\hline 69 & 10 & Elaeocarpus arnhemicus F.Muell. & Elae_ar & & & & $\sqrt{ }$ & & \\
\hline 70 & 11 & Ficus drupacea Thunb. & Ficu_dr & & & & $\sqrt{ }$ & & \\
\hline 71 & 12 & Garcinia $\times$ mangostana $\mathrm{L}$. & Garc_žz̆ & & & & $\sqrt{ }$ & & \\
\hline 72 & 13 & Adenanthera novo-guineensis Baker $\mathrm{f}$. & Aden_no & & & & $\sqrt{ }$ & & \\
\hline 73 & 14 & Sloanea pullei O.C.Schmidt ex A.C.Sm. & Sloa_pu.1 & & & & $\sqrt{ }$ & & \\
\hline 74 & 15 & Calophyllum peekelii Lauterb. & Calo_pe & & & & $\sqrt{ }$ & & \\
\hline 75 & 16 & Cynometra ramiflora $\mathrm{L}$. & Cyno_ra & & & & $\sqrt{ }$ & & \\
\hline 76 & 17 & Dracontomelon dao (Blanco) Merr. \& Rolfe & Drac_da & & & & $\sqrt{ }$ & & \\
\hline 77 & 18 & Prainea limpato (Miq.) Beumee ex K.Heyne & Prai_li & & & & $\sqrt{ }$ & & \\
\hline 78 & 19 & Cleistanthus oblongifolius (Roxb.) Müll.Arg. & Clei_ob & & & & $\sqrt{ }$ & & \\
\hline 79 & 20 & Glochidion sp. & Gloc_sp & & & & $\sqrt{ }$ & & \\
\hline 80 & 21 & Harpullia cupanioides Roxb. & Harp_cu & & & & $\sqrt{ }$ & & \\
\hline 81 & 22 & Pometia pinnata J.R.Forst. \& G.Forst. & Pome_pi & & & & $\sqrt{ }$ & & \\
\hline 82 & 23 & Ficus sp. & Ficu_sp & & & & $\sqrt{ }$ & & \\
\hline 83 & 24 & Pisonia grandis $\mathrm{R} . \mathrm{Br}$. & Piso_gr & & & & $\sqrt{ }$ & & \\
\hline 84 & 1 & Sterculia macrophylla Vent. & Ster_ma & & & & & $\sqrt{ }$ & \\
\hline 85 & 2 & Nageia wallichiana (C.Presl) Kuntze & Nage_wa & & & & & $\sqrt{ }$ & \\
\hline 86 & 3 & Pometia acuminata Radlk. & Pome_ac & & & & & $\sqrt{ }$ & \\
\hline 87 & 4 & Horsfieldia irya (Gaertn.) Warb. & Hors_ir & & & & & $\sqrt{ }$ & \\
\hline 88 & 5 & Canarium birsutum Willd. & Cana_hi & & & & & $\sqrt{ }$ & \\
\hline 89 & 6 & Hopea iriana Slooten & Hope_ir & & & & & $\sqrt{ }$ & \\
\hline 90 & 7 & Elaeocarpus sp. & Elae_sp & & & & & $\sqrt{ }$ & \\
\hline 91 & 8 & Vatica rassak. Blume & Vati_ra & & & & & $\sqrt{ }$ & \\
\hline 92 & 9 & Canarium asperum Benth. & Cana_as & & & & & $\sqrt{ }$ & \\
\hline 93 & 10 & Hopea celtidifolia Kosterm. & Hope_ce & & & & & $\sqrt{ }$ & \\
\hline 94 & 11 & Gymnacranthera farquhariana (Hook.f. \& Thomson) Warb. & Gymn_fa & & & & & $\sqrt{ }$ & \\
\hline 95 & 12 & $\begin{array}{l}\text { Planchonella anteridifera (C.T.White \& W.D.Francis ex Lane-Poole) } \\
\text { H.J.Lam }\end{array}$ & Plan_an & & & & & $\sqrt{ }$ & \\
\hline 96 & 13 & Melicope elleryana (F. Muell.) T.G. Hartley & Meli_el & & & & & $\sqrt{ }$ & \\
\hline 97 & 14 & Anisoptera thurifera subsp. polyandra (Blume) P.S.Ashton & Anis_th & & & & & $\sqrt{ }$ & \\
\hline 98 & 15 & Calopbyllum caudatum Kaneh. \& Hatus. & Calo_ca & & & & & $\sqrt{ }$ & \\
\hline 99 & 16 & Terminalia copelandi Elmer & Term_co.1 & & & & & $\sqrt{ }$ & \\
\hline 100 & 17 & Alstonia spectabilis $\mathrm{R} . \mathrm{Br}$. & Alst_sp & & & & & $\sqrt{ }$ & \\
\hline 101 & 18 & Blumeodendron tokbrai (Blume) Kurz & Blum_to & & & & & $\sqrt{ }$ & \\
\hline 102 & 19 & Sloanea pulchra (Schltr.) A.C.Sm. & Sloa_pu & & & & & $\sqrt{ }$ & \\
\hline 103 & 20 & Garcinia sp. & Garc_sp & & & & & $\sqrt{ }$ & \\
\hline 104 & 21 & Gironniera subaequalis Planch. & Giro_su & & & & & $\sqrt{ }$ & \\
\hline 105 & 22 & Pimelodendron amboinicum Hassk. & Pime_am & & & & & $\sqrt{ }$ & \\
\hline 106 & 23 & Parastemon versteeghii Merr. \& L.M.Perry & Para_ve & & & & & $\sqrt{ }$ & \\
\hline 107 & 24 & Lithocarpus rufovillosus (Markgr.) Rehder & Lith_ru & & & & & $\sqrt{ }$ & \\
\hline 108 & 25 & Sundacarpus amarus (Blume) C.N.Page & Sund_am & & & & & $\sqrt{ }$ & \\
\hline 109 & 26 & Knema sp. & Knem_sp & & & & & $\sqrt{ }$ & \\
\hline 110 & 27 & Endiandra sp. & Endi_sp & & & & & $\sqrt{ }$ & \\
\hline 111 & 28 & Campnosperma brevipetiolatum Volkens & Camp_br & & & & & $\sqrt{ }$ & \\
\hline 112 & 29 & Prunus javanica (Teijsm. \& Binn.) Miq. & Prun_ja & & & & & $\sqrt{ }$ & \\
\hline 113 & 30 & Planchonella keyensis H.J.Lam & Plan_ke & & & & & $\sqrt{ }$ & \\
\hline 114 & 31 & Sysygium anomalum Lauterb. & Syzy_an & & & & & $\sqrt{ }$ & \\
\hline 115 & 32 & Cinnamomum sp. & Cinn_sp & & & & & $\sqrt{ }$ & \\
\hline 116 & 33 & Halfordia kendack Guillaumin & Half_ke & & & & & $\sqrt{ }$ & \\
\hline 117 & 34 & Planchonia careya (F.Muell.) R.Knuth & Plan_ca & & & & & $\sqrt{ }$ & \\
\hline
\end{tabular}


Table 1 Continued

\begin{tabular}{|c|c|c|c|c|c|c|c|c|c|}
\hline \multicolumn{2}{|c|}{ No } & Species & Code & $\mathrm{PF}$ & X1LF & X5LF & $\begin{array}{l}\text { X10LF } \\
\text { X15LF }\end{array}$ & ALL & NON_AC \\
\hline 118 & 35 & Endiandra rubescens (Blume) Miq. & Endi_ru & & & & & $\sqrt{ }$ & \\
\hline 119 & 36 & Homalium foetidum Benth & Homa_fo & & & & & $\sqrt{ }$ & \\
\hline 120 & 37 & Virola surinamensis (Rol. ex Rottb.) Warb. & Viro_su & & & & & $\sqrt{ }$ & \\
\hline 121 & 38 & Cananga odorata (Lam.) Hook.f. \& Thomson & Cana_od & & & & & $\sqrt{ }$ & \\
\hline 122 & 39 & Grewia eriocarpa Juss. & Grew_er & & & & & $\sqrt{ }$ & \\
\hline 123 & 1 & Barringtonia sp. & Barr_sp & & & & & & $\sqrt{ }$ \\
\hline 124 & 2 & Cocblospermum gillivraei Benth. & Coch_gi & & & & & & $\sqrt{ }$ \\
\hline 125 & 3 & Gluta papuana Ding Hou & Glut_pa & & & & & & $\sqrt{ }$ \\
\hline 126 & 4 & Maranthes sp & Mara_sp & & & & & & $\sqrt{ }$ \\
\hline 127 & 5 & Sysygium branderborstii Lauterb. & Syzy_br & & & & & & $\sqrt{ }$ \\
\hline
\end{tabular}

Those species existed in each forest group (unlogged, one year, five years, ten years and fifteen years post selectively-logged). Patterns of tree communities were formed for each forest group, especially for understory mostly occurred after logging activities. Upperstory were mainly recruited from understory of remnant trees. Several upperstory species were present before logging activities occurred in the forests. Our study presented the results of understory and upperstory communities influenced by logging activities and edaphic conditions.

There were three patterns established in our study i.e. 1. tree species formed a tree community in a forest group; 2. tree species present in all forest groups; and 3. tree species did not form a community. Presence of certain tree species as understory in all forest groups was facilitated by ecological alterations, including logging activities. Several tree species existed in all forest groups indicating that those tree species were not influenced by ecological alterations.

Distribution of understory tree community was depicted using CCA having $55.34 \%$ of the variation for two axes; variation for axis 1 was $30 \%$ and variation for axis 2 was $25.34 \%$ (Fig. 3; Table 2). ANOVA showed that the model was significant with $p<0.05$.

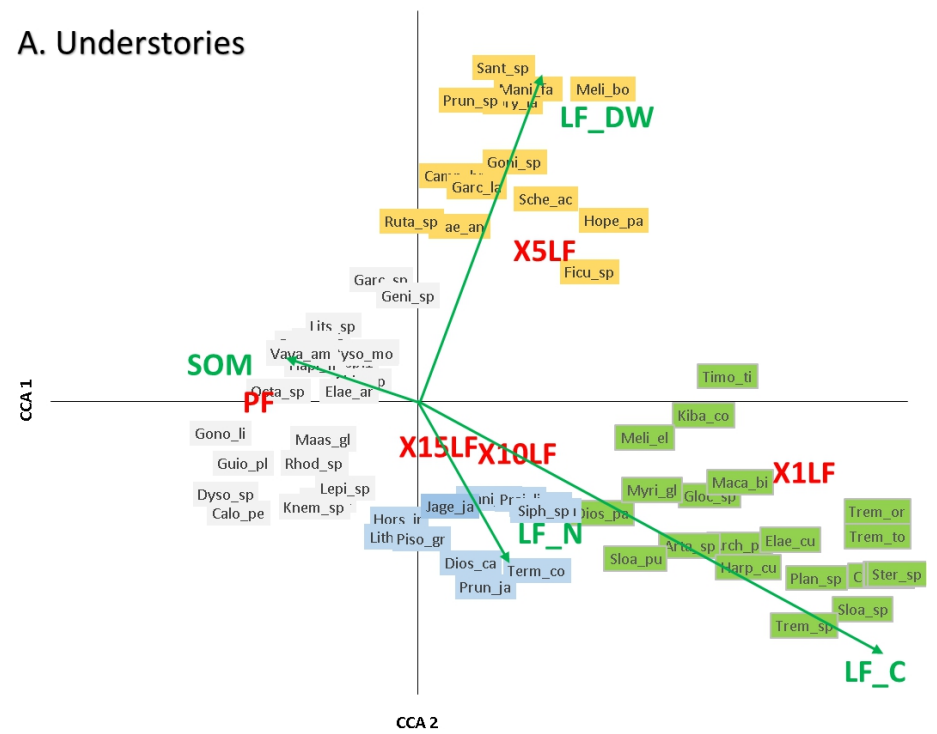

Figure 3 Understory of four tree communities formed due to logging activities symbolized as grey (species grown in PF), green (species grown in X1LF), yellow (species grown in X5LF) and blue (species grown in X10LF-X15LF)

Note: $\mathrm{PF}=$ unlogged forest; $\mathrm{X} 1 \mathrm{LF}=$ one year post selectively-logged forest; X5LF = five years post selectivelylogged forest; X10LF = ten years post selectively-logged forest; X15LF = fifteen years post selectivelylogged forest; SOM = Soil Organic Matter (\%); LF_C = Carbon content in litterfall (\%); LF_N = Nitrogen content in litterfall (\%); LF_DW = dry weight of litterfall $(\mathrm{g})$ 
Table 2 Summary of Canonical Correspondence Analysis (CCA) for understory tree community

\begin{tabular}{lcccc}
\hline \multirow{2}{*}{ Importance of components } & \multicolumn{2}{c}{ Axes } & \multirow{2}{*}{ Total Inertia } \\
\cline { 2 - 3 } & CCA1 & CCA2 & \\
\hline Eigenvalue & 0.2152 & 0.1818 & 0.7175 \\
Proportion explained & 0.3 & 0.2534 & \\
Cumulative proportion & 0.3 & 0.5534 & \\
\hline
\end{tabular}

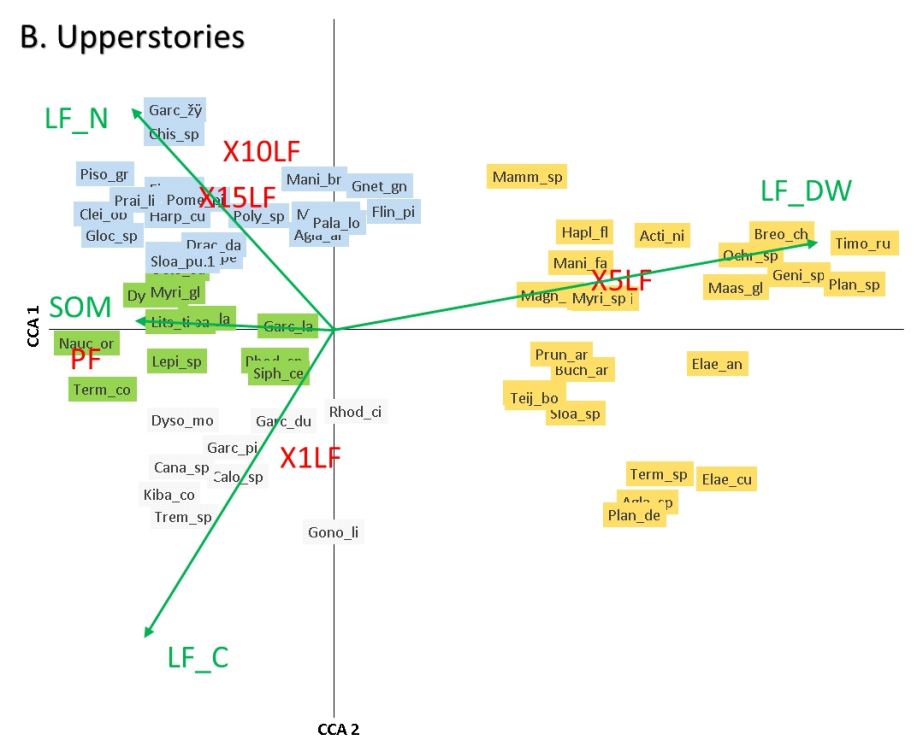

Figure 4 Upperstory of four tree communities formed due to logging activities symbolized as grey (species grown in PF), green (species grown in X1LF), yellow (species grown in X5LF) and blue (species grown in X10LF-X15LF)

Note: $\mathrm{PF}=$ unlogged forest; $\mathrm{X} 1 \mathrm{LF}=$ one year post selectively-logged forest; X5LF = five years post selectivelylogged forest; $\mathrm{X} 10 \mathrm{LF}=$ ten years post selectively-logged forest; $\mathrm{X} 15 \mathrm{LF}=$ fifteen years post selectivelylogged forest; SOM = Soil Organic Matter (\%); LF_C = Carbon content in litterfall (\%); LF_N = Nitrogen content in litterfall (\%); LF_DW = dry weight of litterfall (g)

Table 3 Summary of Canonical Correspondence Analysis (CCA) for upperstory tree community

\begin{tabular}{lccc}
\hline \multirow{2}{*}{ Importance of components } & \multicolumn{2}{c}{ Axes } & \multirow{2}{*}{ Total Inertia } \\
\cline { 2 - 3 } & CCA1 & CCA2 & \multirow{2}{*}{0.6277} \\
\hline Eigenvalue & 0.1961 & 0.1697 & \\
Proportion explained & 0.3124 & 0.2703 & \\
Cumulative proportion & 0.3124 & 0.5826 & \\
\hline
\end{tabular}

Canonical Correspondence Analysis (CCA) grouped the understory tree species into four tree communities i.e. 28 species in the unlogged forest; 21 species in the one year post selectively-logged forest; 21 species in the five years post selectivelylogged forest and 17 species in the ten and fifteen years post selectively-logged forest (Table 1a). Distribution of upperstory tree community was shown of having variation of two axes of $58.26 \%$ with $31.24 \%$ variation for axis 1 and $27.03 \%$ variation for axis 2 (Fig. 4; Table 3). The CCA model was significant at $p<0.05$.

\section{Edaphic Factors}

Interactions among SOM, C content, N content, dry weight of litterfall and forest groups (unlogged, one year, five years, ten years and fifteen years post selectively-logged forests) were analyzed using CCA to figure out the fitting edaphic factors as the indicators of logged forest recovery. Results of CCA showed that SOM tended to be higher in the unlogged forest, dry weight of litterfall tended to be higher in the five years post selectively-logged forest and $\mathrm{C}$ content of litterfall was higher in the one-year post selectively-logged forest (Fig. $3 \& 4$; Table 4).

Based on this analysis, the ten and fifteen years post selectively-logged forests were still in the recovery process, indicated by lower SOM content in those two logged forests compared to 
Table 4 ANOVA of CCA to analyze interactions among SOM, C content, N content, dry weight of litterfall and forest groups (unlogged, one year, five years, ten years and fifteen years post selectively-logged forests)

\begin{tabular}{lcccccc}
\hline \multicolumn{1}{c}{ Edaphic factors } & Df & Sums of square & Mean square & F.Model & $\mathrm{R}^{2}$ & $\mathrm{P}$ \\
\hline SOM & 1 & 0.746 & 0.74644 & 2.438868 & 0.01442 & $0.001 *$ \\
LF_C & 1 & 0.692 & 0.6916 & 2.259688 & 0.01336 & $0.001 *^{*}$ \\
LF_N & 1 & 0.543 & 0.54259 & 1.772822 & 0.01048 & $0.005 *$ \\
LF_DW & 1 & 0.795 & 0.79469 & 2.596517 & 0.01536 & $0.001 *^{*}$ \\
Residuals & 161 & 49.27566 & 0.30606 & & 0.94638 & \\
Total & 165 & 52.05166 & & & & \\
\hline Note: $*$ significant at $p<0.05$ & & & & &
\end{tabular}

the unlogged forest. In contrast, dry weight of litterfall tended to be higher in all logged forests. These results were not in line with research results obtained from logged Bornean rainforest, in which one year post-logged forest produced less litterfall compared to that in the Bornean primary forest. The amount of litterfall in Bornean primary forest was similar to those in the Bornean five years post-logged forest (Prasetyo et al. 2015). This condition suggested that responses of logged forests were depended on ecological circumstances. Furthermore, specific silvicultural treatments should be designed carefully by considering forest condition.

\section{Ecological Changes as a Response to Selective Logging}

Tree communities in the unlogged forest were different from those in the logged forests. The differences were due to ecological changes caused by logging activities resulted in alteration of species composition (Arbainsyah et al. 2014; Verburg \& van Eijk-Bos 2003; Lozada et al. 2012), tree density (Decocq et al. 2014), tree growth rate (Murdjoko et al. 2016b) and association patterns among biotic factors, light availability, ambient moisture, temperature, soil properties and litterfall stock as abiotic factors (Murdjoko et al. 2016c). Tree communities were formed as responses of each tree characteristics toward different ecological circumstances in logged forests. Understory and upperstory tree communities had different reactions toward ecological changes (Murdjoko et al. 2016a; Zhu et al. 2015b). Therefore, there were understory and upperstory tree communities consisted of the same species. Tree communities consisted of seedlings and saplings stages that required more light (Karsten et al. 2014; Flores et al. 2014).
This is the reason why logged forests had altered tree compositions compared to those in the primary forest. Each logged forest has different species composition of the understory tree community. Species composition of the understory tree community was different among the logged forests. Understory tree community in the one year post selectively-logged forest had very different species composition compared to those in the unlogged forest (Fig. 3). Understory tree community in the five years post selectivelylogged forest had very different species composition compared to those in the ten and fifteen years post selectively-logged forests (Fig. 3). These differences in species composition were influenced by changes in environmental conditions (Corrià-Ainslie et al. 2015; Schnitzer \& Walter 2013; Duah-Gyamfi et al. 2014).

The CCA showed that understory tree community in the one year post selectively-logged forest was mainly influenced by Carbon content of litterfall. Understory tree community in the five years post selectively-logged forest was formed as a response toward dry weight of litterfall. The nitrogen content of litterfall affected the establishment of understory tree community in the ten and fifteen years post selectively-logged forests. Understory tree community in the unlogged forest was influenced by SOM content. Alterations of soil characteristics in the logged forests were caused by the change of microclimate conditions (Asase et al. 2014; Imai et al. 2012). Logging activities were responsible for the widening canopy gap leading to the increase of light availability toward understory tree community (Schwartz 2016). Logging activities were also responsible for the decrease of tree density causing the changes in tree growth rates (Verburg \& van Eijk-Bos 2003; Cannon et al. 1998; Do et al. 2016). These 
conditions triggered space and light competitions among tree species, especially in the seedlings and saplings stages(Laurans et al.2014).

Upperstory tree community had different patterns from the understory tree community. In the unlogged forest, species composition of understory was different from that of upperstory tree community. Conspecific association occurred in the unlogged forest. Not all species grown in the understory tree community grew in the upperstory tree community of unlogged forest (Murdjoko et al. 2016a). Ecological condition occurred in the upperstory tree community was similar to that in the understory tree community. Trees in tropical forest experienced more diameter growth in the upperstory tree community (Zhu et al. 2015a). Upperstory tree community in the unlogged forest had very different species composition compared to those in the five years post selectively-logged forest (Fig. 4). However, similar species composition was observed among upperstory tree communities in the unlogged forest, one year post selectivelylogged forest, ten and fifteen years post selectively-logged forests (Fig. 4). Tree species located in the five years post selectively-logged forest was the results of species competition caused by the change of ecological conditions. Thus, the current species were not the same as the previous species because of the duration of the ecological process. Upperstory tree community in the logged forests showed a dynamic establishment of tree community. Each species had different growth rate as a response to logging impact (Murdjoko et al. 2016b). Some species had higher population growth rate than others leading to higher survival rate (Murdjoko 2013; Zuidema et al. 2009). Although recovery process was seen to be happening in the ten and fifteen years post selectively-logged forests, the process still requires more time to reach the fully recovered stage.

\section{Implication of Ecological Approach for Sustainable Forest Management}

This study proposed an ecological approach to determine whether logged forests were recovered in fifteen years. Existing tree communities and edaphic factors, especially SOM, in the unlogged forest were used as a reference of logged forest reaching recovered condition. SOM plays an important role to support nutrient absorption in soil (Mutiso et al. 2013). The soil of South Papua is mainly classified as Ultisols, so the characteristic of soil is infertile (Marshall \& Beehler 2012). Selective logging activities did not seem to totally change ecological condition. The logged forest was declared to be fully recovered when its conditions had reached similar condition as those in unlogged (primary) forest, especially in terms of ecological aspects such as the content of SOM, stem density and species composition. Therefore, it is imperative to set permanent sample plots in the unlogged (primary) and logged forests, to conduct intensive and persistent monitoring of ecological conditions and tree growth (Krisnawati \& Wahjono 2010; Ruslandi et al. 2017a; Ruslandi et al. 2017b). The monitoring results would be valuable as basic information to further evaluate the silviculture protocol. Useful modifications could be designed by taking ecological perspective into account.

\section{CONCLUSIONS}

Understory and upperstory tree communities formed different patterns due to logging activities. Species composition existed in the tree communities in the ten and fifteen years post selectively-logged forests were not similar to that in the unlogged forest, meaning that the logged forests were still in the recovery process. SOM content in the logged forest was lower compared to that in the unlogged forest, indicating that the logged forests were not fully recovered. These occurrences indicated that it took more than fifteen years for the logged forests to be fully recovered. Long-term studies are necessary to continuously monitor the ecological process in the logged forest in reaching the recovery stage. The recorded influential ecological factors obtained from this study can be used as indicators for logged forest recovery.

\section{ACKNOWLEDGEMENTS}

The research was funded by Beasiswa Pendidikan Pascasarjana Dalam Negeri (BPPDN) 2014, Manokwari Regency and West Papua Province. The authors are thankful to the 
following people for assisting in fieldwork and species vegetation identification as well as providing useful inputs: Nithanel M. H. Benu (Balai Penelitian dan Pengembangan Lingkungan Hidup dan Kehutanan Manokwari), Dr Purnomo (Faculty of Biology, Gadjah Mada University), Prof Dr Ir Suryo Hardiwinoto, M. Agr. (Faculty of Forestry, Gadjah Mada University), Dr Sena Adi Subrata, M.Sc (Faculty of Forestry, Gadjah Mada University), Dr Ir Soewarno Hasanbahri, M.S. (Faculty of Forestry, Gadjah Mada University), Dr Ir Eko Hanudin, M.Sc (Faculty of Agriculture, Gadjah Mada University), Prof Dr Charlie Danny Heatubun, S.Hut, M.Si, FLS (Faculty of Forestry, Papua University), Dr Ir Relawan Kuswandi, M.Sc, Daud Bano, Dedy Subrata May, Vinoba Chandra, Ari Wibowo, Anton Bongga, Sri Emy Rahayu and all staff of PT Tunas Timber Lestari. We are also grateful to anonymous reviewers for valuable comments .

\section{REFERENCES}

Arbainsyah, de Iongh HH, Kustiawan W, de Snoo GR. 2014. Structure, composition and diversity of plant communities in FSC-certified, selectively logged forests of different ages compared to primary rain forest. Biodivers Conserv 23:2445-72.

Asase A, Asiatokor BK, Ofori-Frimpong K. 2014. Effects of selective logging on tree diversity and some soil characteristics in a tropical forest in Southwest Ghana. J For Res 25:171-6.

Cannon CH, Peart DR, Leighton M. 1998. Tree species diversity in commercially logged Bornean rainforest. Science 80:1366-8.

Core Team R. 2014. R: A language and environment for statistical computing. Vienna (AT): R Foundation for Statistical Computing.

Corrià-Ainslie R, Julio-Camarero J, Toledo M. 2015. Environmental heterogeneity and dispersal processes influence post-logging seedling establishment in a Chiquitano dry tropical forest. For Ecol Manage 349:122-33.

Decocq G, Beina D, Jamoneau A, Gourlet-Fleury S, ClossetKopp D. 2014. Don't miss the forest for the trees! Evidence for vertical differences in the response of plant diversity to disturbance in a tropical rain forest. Perspect Plant Ecol Evol Syst 16:279-87.

Do TV, Cam NV, Sato T, Binh NT, Kozan O, Thang NT, Mitlöhner R. 2016. Post-logging regeneration and growth of commercially valuable tree species in evergreen broadleaf forest. Vietnam. J Trop For Sci 28:426-35.

Duah-Gyamfi A, Swaine EK, Adam KA, Pinard MA, Swaine MD. 2014. Can harvesting for timber in tropical forest enhance timber tree regeneration? For Ecol Manage 314:26-37.

Edwards DP, Tobias JA, Sheil D, Meijaard E, Laurance WF. 2014. Maintaining ecosystem function and services in logged tropical forests. Trends Ecol Evol 29:511-20.

Flores O, Hérault B, Delcamp M, Garnier É, GourletFreury S. 2014. Functional traits help predict postdisturbance demography of tropical trees PLoS ONE 9(9): e105022. Available from: https://doi.org/10.1371/journal.pone.010502.

Gandhi Y, Mitlöhner R. 2014. Tree species composition, diversity and structure in Tunas logging concession area of Papua-Indonesia. Tree 66:47.

Hattori D, Kenzo T, Irino KO, Kendawang JJ, Ninomiya I, Sakurai K. 2013. Effects of soil compaction on the growth and mortality of planted Dipterocarp seedlings in a logged-over tropical rainforest in Sarawak, Malaysia. For Ecol Manage 310:770-6.

Hendri, Yamashita T, Kuntoro AA, Lee HS. 2012. Carbon stock measurements of a degraded tropical loggedover secondary forest in Manokwari Regency, West Papua, Indonesia. For Stud China 14:8-19.

Hoang VS, Baas P, Keßler PJA, Slik JWF, Steege HT, Raes N. 2011. Human and environmental influences on plant diversity and composition in Ben En National Park, Vietnam. J Trop For Sci 23:328-37.

Imai N, Kitayama K, Titin J. 2012. Effects of logging on phosphorus pools in a tropical rainforest of Borneo. J Trop For Sci 24:5-17.

Karsten RJ, Meilby H, Larsen JB. 2014. Regeneration and management of lesser known timber species in the Peruvian Amazon following disturbance by logging. For Ecol Manage 327:76-85.

Khairil M, Wan Juliana WA, Nizam MS. 2014. Edaphic influences on tree species composition and community structure in a tropical watershed forest in Peninsular Malaysia. J Trop For Sci 26:284-94.

Krisnawati H, Wahjono D. 2010. Effect of post-logging silvicultural treatment on growth rates of residual stand in a tropical forest. Indones J For Res 2:112-24.

Kuswandi R, Murdjoko A. 2015. Population structures of four tree species in logged-over tropical forest in South Papua, Indonesia: an integral projection model approach. Indones J For Res 2:93-101.

Kuswandi R, Sadono R, Supriyatno N, Marsono D. 2015. Keanekaragaman struktur tegakan hutan alam bekas tebangan berdasarkan biogeografi di Papua. J Mns dan Lingkung 22:151-9. 
Kuswandi R. 2014. The effect of silvicultural treatment on stand growth of logged-over forest in South Papua. Indones J For Res 1:117-26.

Laurans M, Hérault B, Vieilledent G, Vincent G. 2014. Vertical stratification reduces competition for light in dense tropical forests. For Ecol Manage 329:79-88.

Lozada J, Arends E, Sánchez D, Villarreal A, Soriano P, Costa M. 2012. Vegetation succession of logged forest in the western alluvial plains of vegetation succession of logged forest in the Western Alluvial Plains of Venezuela. J Trop For Sci 24:300-11.

Marshall AJ, Beehler BM. 2012. The ecology of Papua: Part one. Hongkong (HK): Periplus Editions. p. 168-9.

Murdjoko A. 2013. Recuperation of non-commercial trees in logged forest in Southern Papua, Indonesia. J Man Hut Trop 19:94-102.

Murdjoko A, Marsono D, Sadono R, Hadisusanto S. 2016 a. Plant species composition and their conspecific association in natural tropical rainforest, South Papua. Biosaintifika J Biol Biol Educ 8:33-46.

Murdjoko A, Marsono D, Sadono R, Hadisusanto S. 2016b. Population dynamics of Pometia for the period of post-selective logging in tropical rainforest, Southern Papua, Indonesia. Biosaintifika J Biol Biol Educ 8:321-30.

Murdjoko A, Marsono D, Sadono R, Hadisusanto S. $2016 \mathrm{c}$. Tree association with Pometia and its structure in logging concession of South Papua Forest. J Man Hut Trop 22:180-91.

Mutiso FM, Hitimana J, Kiyiapi JL, Sang FK, Eboh E. 2013. Recovery of Kakamega Tropical Rainforest from anthropogenic disturbances. J Trop For Sci 25:566-76.

Oksanen J, Blanchet FG, Kindt R, Legendre P, Minchin PR, O'Hara RB, ... Oksanen MJ. 2013. Package 'vegan'. Community ecology package, version, 2(9).

Pennington RT, Hughes M, Moonlight PW. 2015. The origins of tropical rainforest hyperdiversity. Trends Plant Sci 20:693-5.

Petocz RG. 1989. Conservation and development in Irian Jaya: a strategy for rational resource utilization. Leiden (NL): E. J. Brill.

Prasetyo E, Hardiwinoto S, Supriyo H. 2015. Litter production of logged-over forest using Indonesia selective cutting system and strip planting (TPTJ) at PT Sari Bumi Kusuma. Procedia Environ Sci 28:676-82.

Putz FE, Romero C. 2014. Futures of tropical forests (sensu lato). Biotropica 46:495-505.

Ruslandi R, Cropper Jr. WP, Putz FE. 2017a. Effects of silvicultural intensification on timber yields, carbon dynamics, and tree species composition in a
Dipterocarp forest in Kalimantan, Indonesia: an individual-tree-based model simulation. For Ecol Manage 390:104-18.

Ruslandi R, Cropper Jr. WP, Putz FE. 2017b. Tree diameter increments following silvicultural treatments in a Dipterocarp forest in Kalimantan, Indonesia : a mixed-effects modelling approach. For Ecol Manage 396:195-206.

Rutten G, Ensslin A, Hemp A, Fischer M. 2015. Forest structure and composition of previously selectively logged and non-logged montane forests at Mt. Kilimanjaro. For Ecol Manage 337:61-6.

Schnitzer SA, Walter PC. 2013. Treefall gaps and the maintenance of species diversity in a tropical forest. Ecology 82:913-9.

Schwartz G. 2016. Profitability of silvicultural treatments in logging gaps in the Brazilian Amazon. J Trop For Sci 28:68-78.

Sist P, Mazzei L, Blanc L, Rutishauser E. 2014. Large trees as key elements of carbon storage and dynamics after selective logging in the Eastern Amazon. For Ecol Manage 318:103-9.

Susanty FH, Suhendang E, Jaya INS. 2015. Mortality and ingrowth pattern of Dipterocarps in forest recovery in East Kalimantan. BIOTROPIA 22(1):11-23.

ter Braak CJF. 1986. Canonical correspondence analysis: a new eigenvector technique for multivariate direct gradient analysis. Ecology 67:1167-79.

ter Braak CJF. 1987. The analysis of vegetationenvironment relationships by Canonical Correspondence Analysis. Vegetatio 69:69-77.

Verburg R, van Eijk-Bos C. 2003. Effects of selective logging on tree diversity, composition and plant functional type patterns in a Bornean rainforest. J Veg Sci 14:99-110.

Wasrin UR, Putera AE. 1999. Litterfall in a primary and two logged-over lowland tropical rainforests in Pasirmayang, Jambi. BIOTROPIA 14:36-51.

West TAP, Vidal E, Putz FE. 2014. Forest biomass recovery after conventional and reduced-impact logging in Amazonian Brazil. For Ecol Manage 314:59-63.

Whitfeld TJS, Lasky JR, Damas K, Sosanika G, Molem K, Montgomery RA. 2014. Species richness, forest structure, and functional diversity during succession in the New Guinea Lowlands. Biotropica 46:538-48.

Zambrano J, Coates R, Howe HF. 2014. Effects of forest fragmentation on the recruitment success of the tropical tree Poulsenia armata at Los Tuxtlas, Veracruz, Mexico. J Trop Ecol 30:209-18.

Zhu H, Yong C, Zhou S, Wang H, Yan L. 2015a. Vegetation, floristic composition and species diversity in a tropical mountain nature reserve in southern 
Yunnan, SW China, with implications for conservation. Trop Conserv Sci 8:528-46.

Zhu Y, Comita LS, Hubbell SP, Ma K. 2015b. Conspecific and phylogenetic density-dependent survival differs across life stages in a tropical forest. J Ecol 103:957-66.
Zuidema PA, Brienen RJ, During HJ, Güneralp B. 2009. Do persistently fast-growing juveniles contribute disproportionately to population growth? A new analysis tool for matrix models and its application to rainforest trees. Am Nat 174:709-19. 